\title{
The Development and Evaluation of New Measuring Methods of the Adhesion Force of Single Particles
}

\author{
Genji Jimbo* and Ryohei Yamazaki* \\ Department of Chemical Engineering, \\ Nagoya University
}

\begin{abstract}
To make clear the problems in the measurement of the adhesion force of powder, new measuring principles and methods were developed, in which emphasis was laid on extending measuring ranges especially to high temperature condition.

As measuring methods for the adhesion force of single particles, vibration and impaction separation methods were developed, and it was found that in separating a particle, the rolling motion by tangential force was very important, for which a fundamental equation was derived. The application of this tangential force to the adhesion measurement was proposed. Some additional measurements were carried out at high temperature to investigate the effect of temperature on the adhesion force of single particles.
\end{abstract}

\section{Introduction}

A large number of phenomena concerning particulate materials are still unexplained and the reason for the present great dependence on the experiences in making use of the phenomena or in solving the problems caused by them is the poor knowledge about the fundamental dynamic properties of the particulate materials. In spite of the active researches and developments of the measuring methods for the adhesion, agglomeration, friction property, flowability etc, of the powder for these 20 years, especially these 5 years, it is still difficult to say that they provide enough accuracy, reproductivity and exchangeability of the data among them. Above all, with regard to the adhesion force of powder, the measured values are extremely different depending upon the measuring method, as the authors have reported previously. ${ }^{1)}$ Some examples gave the difference of the adhesion force of a single particle in the range of the seventh power of ten.

On the other hand, the necessity for the

\footnotetext{
* Furo-cho, Chigusa-ku, Nagoya, Aichi, 464 TEL. 052 (781) 5111

Received April 25, 1983
}

measurement of the adhesion force shows increasing variety including that at high temperature in the atmosphere of various gases or under the condition with mass transfer and chemical reactions, especially owing to the recent rapid development of new energy.

An improved measuring method has been developed to obtain the adhesion force which is regarded as an important fundamental physical property of powder. In this report, it was attempted to investigate the reasons for the variance of the measured data obtained by the various conventional methods and to extend the conditions for the measurement and the measuring range to a great extent by using the newly developed method.

\section{Principles of measuring methods for ad- hesion force of single particles}

The simplest method for the measurement of the adhesion force of single particles is performed separating the particles from solid surface in the normal direction to the contacting surface by some force. The features of the practical separation methods where the gravity, centrifugal and inertia forces working on the particles are mainly made use of for the separation, are summarized in Table 1 . 
Table 1. The characteristics of various kinds of fundamental measuring methods of adhesion force of single particles

\begin{tabular}{l|l}
\hline \multicolumn{1}{c|}{ Principle } & \multicolumn{1}{c}{ Characteristics and problems } \\
\hline $\begin{array}{l}\text { Centrifugal separation } \\
\text { method }^{2) *}\end{array}$ & $\begin{array}{l}\text { Almost established. } \\
\text { Accurate but expensive. } \\
\text { Difficult in high temperature condition. }\end{array}$ \\
\hline $\begin{array}{l}\text { Vibration separation } \\
\text { method }^{3), 4) * *}\end{array}$ & $\begin{array}{l}\text { Simple but less accurate. } \\
\text { Only in the range of small force. }\end{array}$ \\
\hline $\begin{array}{l}\text { Impaction separation } \\
\text { method }^{5 * *}\end{array}$ & $\begin{array}{l}\text { Difficult in the measurement of separation force. } \\
\text { Less expensive. } \\
\text { Easy to apply to high temperature case. }\end{array}$ \\
\hline
\end{tabular}

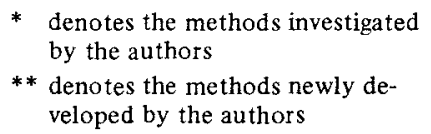

Although the centrifugal separation method developed earliest among them is well known as a standardized one, it is disadvantageous as a general routine method because of the equipment's cost and its operational procedure and besides it is not suitable for the measurement at high temperature and under other special conditions. For these reasons, a vibration and an impaction separation method were tried to develop in order to increase the reliability of the measured adhesion force of a single particle by comparison of data and to make possible the measurement under special conditions including high temperature.

3. Measurement of adhesion force by impaction separation method

\section{1 Measuring method and experimental ap- paratus}

As shown in Fig. 1, particles are dispersed and adhere to a plate (usually glass plate) which is fixed onto a base. By giving the base one-

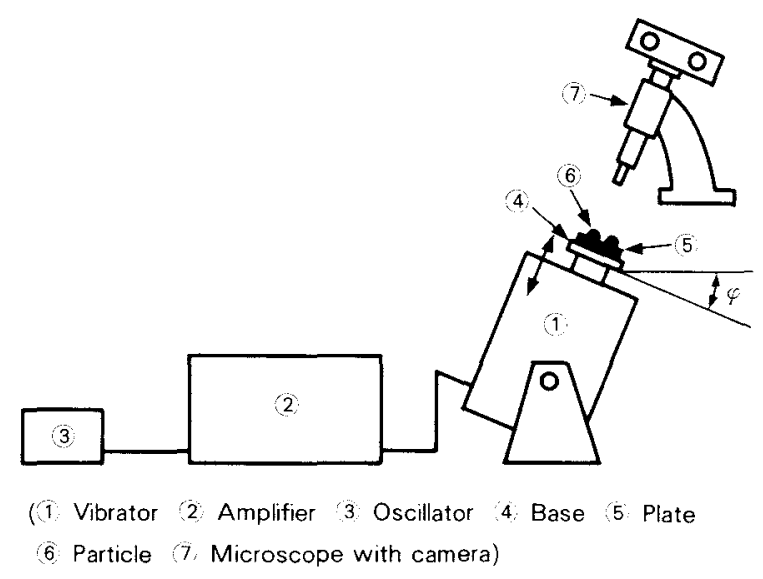

Fig. 1 The exprimental apparatus of vibration separation method dimensional vibration, some of the particles will be separated from the plate by separation force caused by the vibration. The proceeding of the separation is analysed by micrographs which are taken before and after the separation. The adhesion force is supposed to be equal to the separation force $m \alpha \omega^{2}$ where the weight of a particle $m$ is calculated from the particle size. In this case, the vibration base should be inclined with a little angle so that the once separated particles may leave the plate swiftly.

The authors have found that the adhesion forces obtained by the vibration method with a simple modified vibration feeder were less than the data measured by the centrifugal method and that the fact was related to the inclination of the plate and the shape of vibration. Therefore, in the present experiments, a magnetic vibrator (Node made, Model VT-25) was used to produce strictly one-dimensional vibration and the inclination $\varphi$ was exactly set.

\section{2 Measured results}

Some examples of the adhesion force measured by the vibration separation method are shown in Figs. 2 and 3. The glass beads adopted here were Toshiba glass beads GB703K, from which only the beads blocking 145 mesh screen were used for the measurement. The glass plate applied was micro slide glass (Matsunami Glass Industries, Ltd.) with the 1st grade after JIS R3703.

In Figs. 2 and 3 , the separation force $F_{s}$ is taken on the abscissa and the residual percentage of particles on the ordinate. As reported previously ${ }^{2)}$, the relation described by the following logarithmic-normal distribution function has been found between the separation force and the residual percentage of particles. 


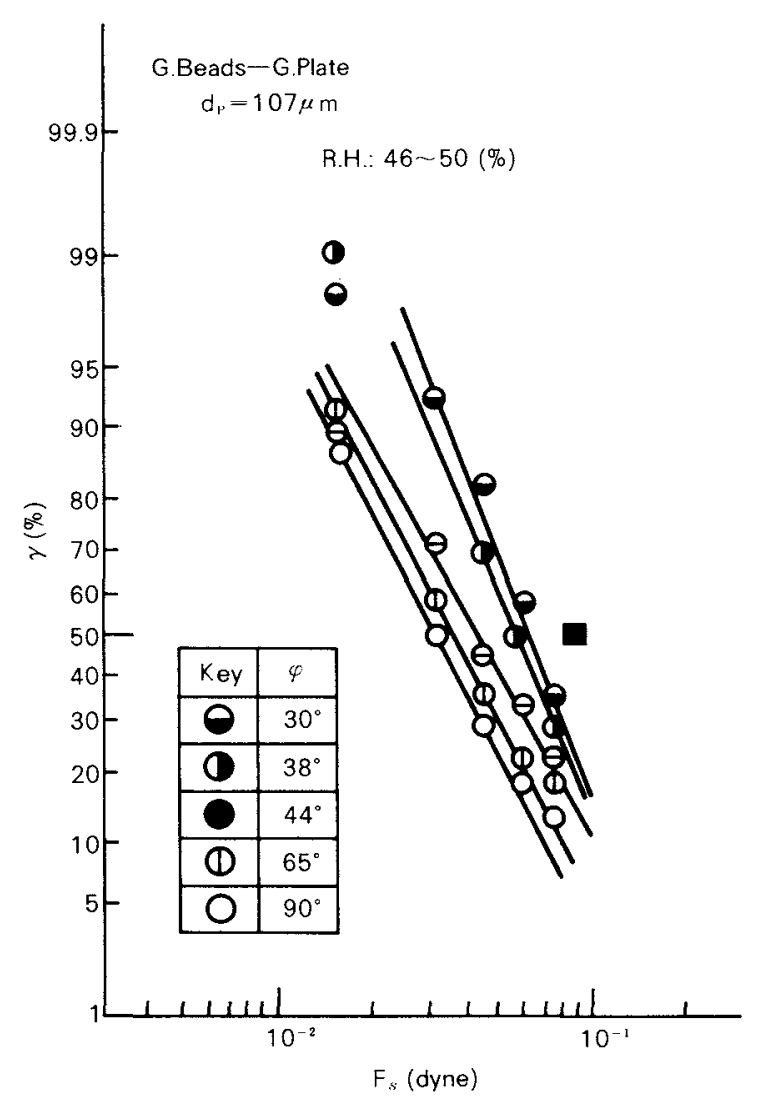

Fig. 2 The results of measurement of glass beads by vibration separation method (normal separation)

$$
\begin{aligned}
\gamma= & \frac{100}{\sqrt{2 \pi} \log \sigma} \int \exp \left[\frac{\left(\log F_{s}-\log \bar{F}_{s}\right)^{2}}{2 \log ^{2} \sigma}\right] \\
& \times d\left(\log F_{s}\right)
\end{aligned}
$$

The effect of the inclination of the adhesion plate shown in Fig. 2 and the temperature effect in Fig. 3 will be discussed in the following section and in chapter 5 , respectively.

\section{3 Discussion on the results}

From the results obtained by the vibration method, some problems can be noticed with regard to the measurement of adhesion force by this method. First, it is generally difficult to give particles a large separation force with exactly one-dimensional vibration as seen in Fig. 3. Especially, when a heating device is attached to the vibrator, the intensity of the vibration is further reduced. Therefore in case large separation force is required, this vibration method is not suitable. Secondly, the adhesion force measured by this method was much smaller than that obtained by the centrifugal method as seen in Fig. 2, where the point with

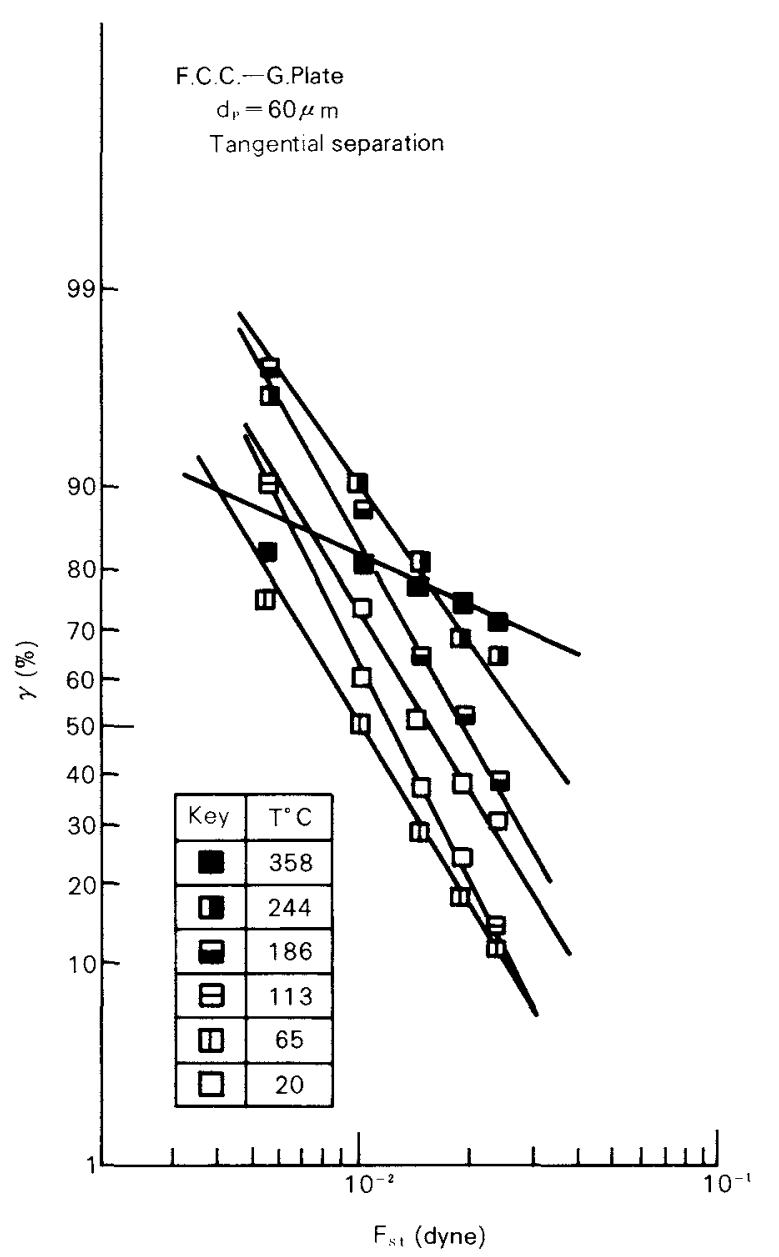

Fig. 3 The results of measurement of F.C.C. Particles by vibration separation method (tangential separation)

a symbol $\boldsymbol{m}$ is the value by the centrifugal method. It was also found that the difference was affected by the inclination $\varphi$.

\section{4 Analysis of measured adhesion force using a rolling model}

The reduction of the adhesion force measured by the vibration method was tried to explain using a following rolling model.

A particle is supposed to adhere to a material with some contacting plane which can be approximated as a flat circle, as shown in Fig. 4. When a separation force is given on the particle by vibration, the normal component of the separation force $F_{s n}$ acts to separate the particle from the contacting plane and at the same time the tangential component $F_{s t}$ acts to separate the particle by rotation around the bottom edge of the contacting plane $H$ in general cases, because it is difficult to give the vibration in 


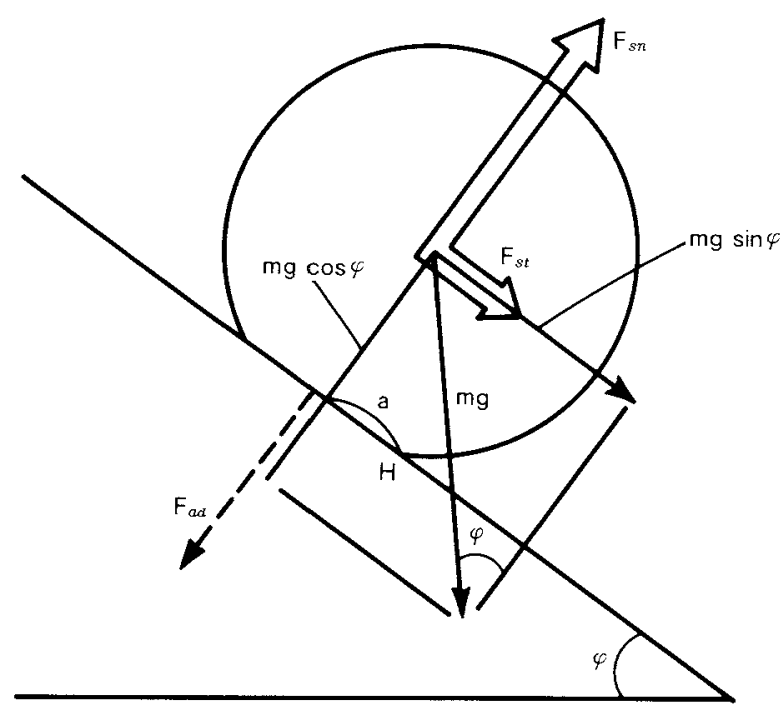

Fig. 4 The model of separation of a single particle from a plane surface.

the exactly vertical direction.

The momental balance around the point $H$ is expressed by the following equation taking all these forces into consideration, where the adhesion force per unit area $f_{a d}$ is supposed to work uniformly over the contacting plane.

$$
\begin{aligned}
& a F_{s n}+1 / 2\left(\sqrt{d_{p}^{2}-4 a^{2}}\right) \mathrm{mg} \sin \varphi+ \\
& +1 / 2\left(\sqrt{\left.d_{p}^{2}-4 a^{2}\right)} F_{s t}=\pi a^{3} f_{a d}+\mathrm{amg} \cos \varphi\right.
\end{aligned}
$$

From the above equation, the separation force $F_{s n}$ is obtained as follows.

$$
\begin{aligned}
F_{s n}= & \pi a^{2} f_{a d}+\mathrm{mg}\left[\cos \varphi-\left(\sqrt{\left(d_{p} / 2 a\right)^{2}-1}\right)\right. \\
& \times \sin \varphi]-\left(\sqrt{\left(d_{p} / 2 a\right)^{2}-1}\right) F_{s t} \\
= & F_{a d}+(\pi / 6) d_{p}^{3} \rho_{s} g\left[\cos \varphi-\left(\sqrt{\left(d_{p} / 2 a\right)^{2}-1}\right)\right. \\
& \times \sin \varphi]-\left(\sqrt{\left(d_{p} / 2 a\right)^{2}-1}\right) F_{s t}
\end{aligned}
$$

This equation shows that the separation force $F_{s n}$ is the adhesion force $F_{a d}$ added by the forces implied in the 2nd and 3rd terms of the right hand side, which are usually negative.

With the reasonable assumptions of $d_{p} \gg a$, therefore

$$
\sqrt{\left(d_{p} / 2 a\right)^{2}-1} \fallingdotseq d_{p} / 2 a
$$

and $\left(d_{p} / a\right) \sin \varphi \gg \cos \varphi$, the equation (3) comes to the next equation.

$$
\begin{aligned}
F_{s n} \fallingdotseq & F_{a d}-(\pi / 6) d_{p}{ }^{3} \rho_{s} g\left(d_{p} / 2 a\right) \sin \varphi \\
& -\left(d_{p} / 2 a\right) F_{s t}
\end{aligned}
$$

As seen in this equation, the tangential component of the separation force $F_{s t}$ multipiled by $d_{p} / 2 a$, which is about 30 as discussed later, has the same magnitude as the normal component $F_{s n}$. This is the reason for the vibration to be required strictly one-dimensional.

(1) When only the effect of inclination is considered

As the tangential component of the vibration is neglected in this case,

$$
F_{s}=F_{a d}-(\pi / 6) d_{p}{ }^{3} \rho_{s} g\left(d_{p} / 2 a\right) \sin \varphi
$$

The data shown in Fig. 2 are plotted in Fig. 5 which confirms the relation implied by the equation (5). From the gradient of the straight line in this figure and the equation (5), the radius of the contacting surface is calculated to be about $1.4 \mu \mathrm{m}$.

The contacting condition may be expressed in the following Hertz's equation taking the pressure caused by the self weight and the adhesion force into consideration.

$$
\left(a / d_{p}\right)^{3}=(3 / 4)\left(\mathrm{mg} / d_{p}^{2}\right)(1-\nu) / E
$$

From the equation with the practical data for glass ( $\nu=0.2, E=8,000 \mathrm{~kg} / \mathrm{mm}^{2}$ ), the value $a$ of deformation by self weight comes to about $230 \AA$ for a particle of $d_{p}=107 \mu \mathrm{m}$. It is much smaller even added by the adhesion force than $1.4 \mu \mathrm{m}$ obtained from the equation (5) and data.

Then the scanning microscopic photograph of the surface of a glass sphere showed the

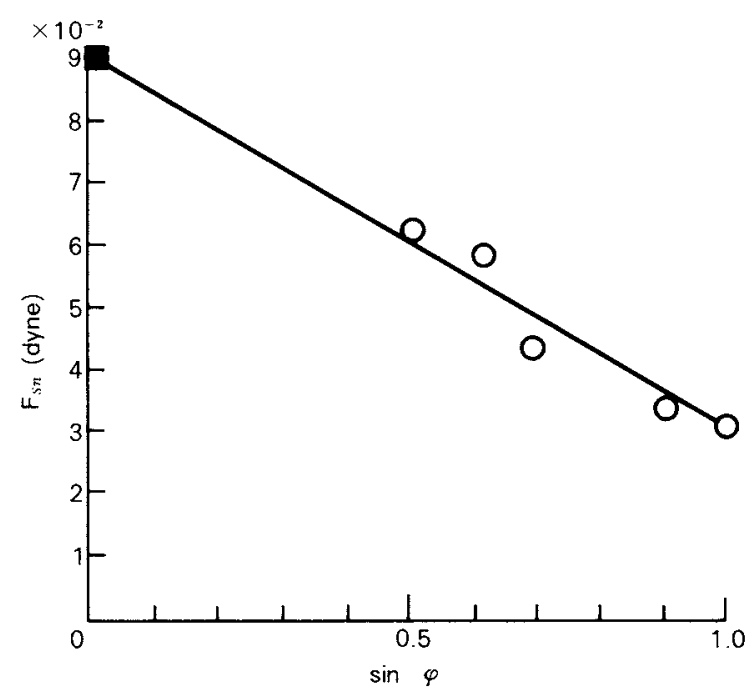

Fig. 5 Relation between separation force $F_{s n}$ and inclination $\varphi$ with a point by centrifugal method 
roughness of about $5 \mu \mathrm{m}$. On the other hand, the calculation of the imaginary contacting circle for the stablest position gave the radius of $3.2 \mu \mathrm{m}$ (in case $d_{p}=107 \mu \mathrm{m}$ ) and $2.5 \mu \mathrm{m}$ (in case $\left.d_{p}=61 \mu \mathrm{m}\right)$ with the assumption of the roughness of $0.1 \mu \mathrm{m}$ for the surface of glass beads which was based on the roughness of glass slide surface according to the maker's information.

From these results it was made clear that the glass bead had a contact area having a diameter of a few $\mu \mathrm{m}$ with the plate by some reason and that the particle can be separated with a much smaller force to cause its rotation than the force working merely in the normal direction. Assuming the relation expressed by equation (5), the adhesion force of single particles $F_{a d}$ can be estimated from the intercept of ordinate at $\varphi=0$.

(2) Discussion on tangential separation method making use of tangential component of vibration

As mentioned in the previous section, the tangential component of vibration is very effective to separate the particles and so it may be possibly made use of for the measurement of adhesion force. Especially, when the separation force is not strong enough, this tangential separation method seems very useful.

In this case, where $F_{s n}=0$ and $\varphi=90^{\circ}$,

$$
F_{s t}=\left(2 a / d_{p}\right) F_{a d}-(\pi / 6) d_{p}{ }^{3} \rho_{s} g
$$

As this method is used in case the particles adhere to a vertical plate, namely $F_{s t} \gg \mathrm{mg}=$ $(\pi / 6) d_{p}{ }^{3} \rho_{s} g$, therefore

$$
F_{s t} \fallingdotseq\left(2 a / d_{p}\right) F_{a d}
$$

This means that the vibration force required to separate a particle is only a few $\%$ of the adhesion force working on it. It would be still less, as the particle weight is subtracted as seen in equation (7).

For the centrifugal separation method to separate a particle in the tangential direction, the equation (8) is obtained by in troducing the relations $F_{s n}=0$ and $\sin \varphi=0$ into the fundamental equation (4). This equation was derived using a different method by Polke. ${ }^{6)}$

In order to confirm this relation, the adhesion force was measured by the tangential vibration method with a newly developed ap-

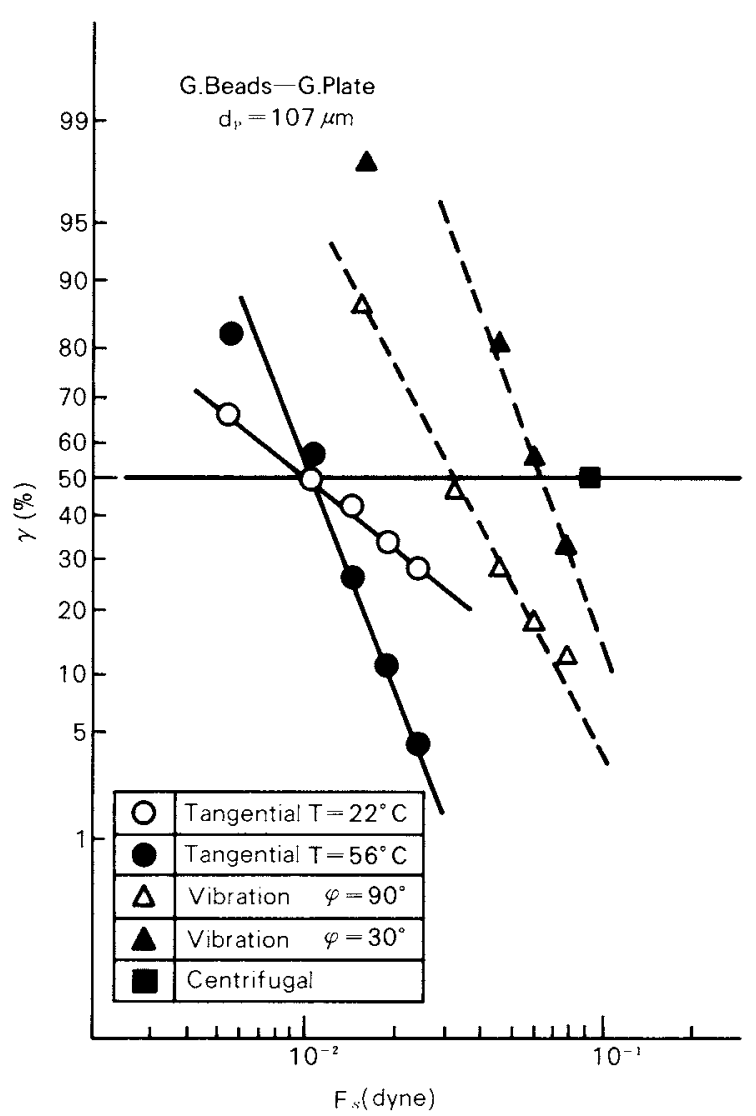

Fig. 6 The results by tangential separation method, with the results by other methods.

paratus. Some examples of the results depicted in Fig. 6 show that they are smaller not only than the data obtained by the centrifugal method (marked with - ) but also than that by the normal vibration method.

Regarding the separation force by the centrifugal method as $F_{a d}$ and using the $50 \%$ - value of the one obtained by the tangential method at $22^{\circ} \mathrm{C}$ and $56^{\circ} \mathrm{C}$ in Fig. 6 for $F_{s t}$, the calculation of $a$ from the equation (7) gave $5.6 \mu \mathrm{m}$. It is nearly 4 times as large as $1.4 \mu \mathrm{m}$ obtained in the previous section but seems reasonable considering the accuracy of the present experiments.

Therefore the tangential vibration method has a great chance for the measurement of the adhesion force of a single particle, when the contacting conditions of the particle can be made clear quantitatively.

4. Measurement of adhesion force by impaction separation method

\section{1 Measuring method and experimental ap- paratus}




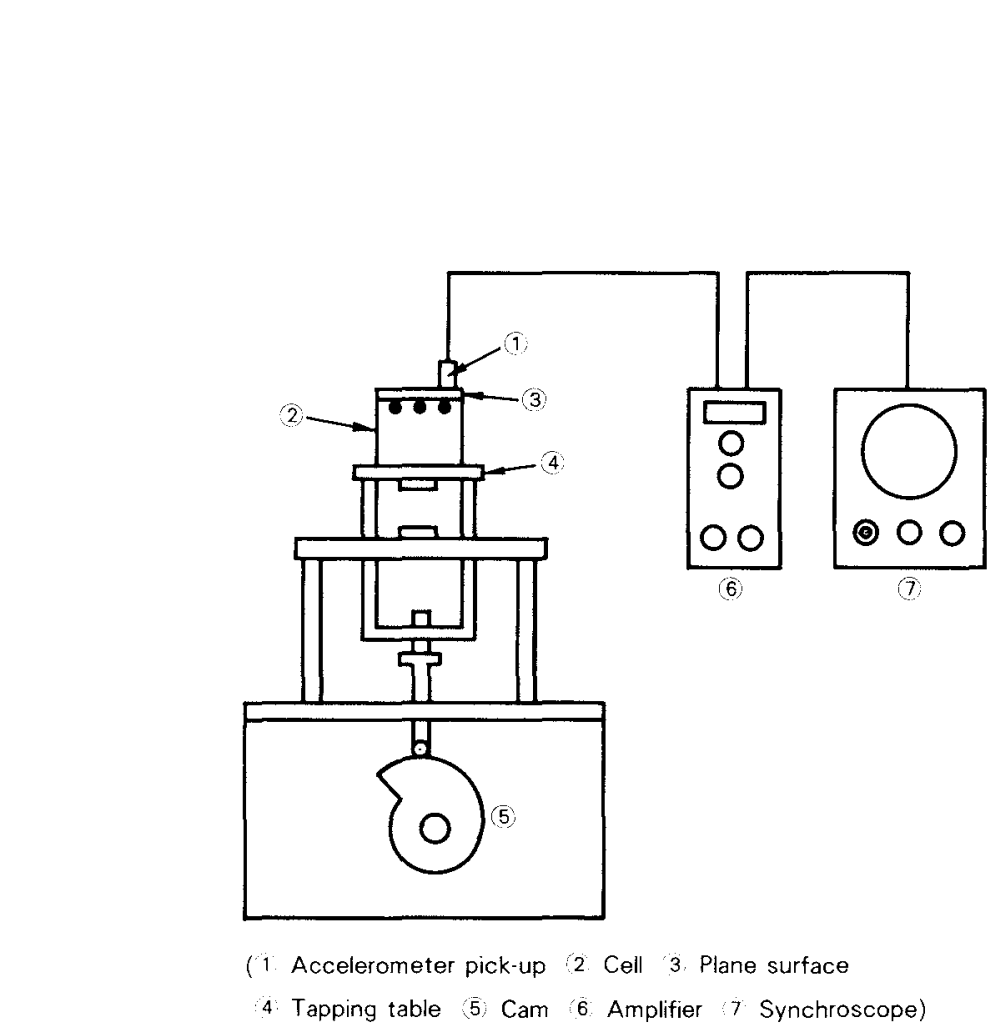

Fig. 7 The experimental apparatus of impaction separation method

In order to extend the measuring range of the adhesion force which was limited in case of the vibration separation method as mentioned above, an impact separation method was developed using a measuring apparatus for which so-called tapping equipment for packing was reconstructed as shown in Fig. 7.

In this case, the acceleration of the particle was measured by the accelerometer pick-up (EMIC made, 509 - CA type) fixed on the top of the measuring cell. As it changed complicatedly with time, the data was taken as the maximum value in the beginning period of the impaction, which would require further investigation. The size of the separated particles and the residual percentage of particles were obtained by the micrographs before and after impaction as above.

\section{Results and discussions}

In Fig. 8, the data obtained by the normal and the tangential impaction method are potted with those by the tangential vibration method. In case the acceleration is given in the tangential direction, the data by the impaction method agree with those by the vibration one very well, while the impaction separation method in the normal direction gives a few times as large data as those by the centrifugal method.

Using equation (7) with the above data by different separation methods, the radius of the contacting plane can be estimated to be $6.9 \mu \mathrm{m}$

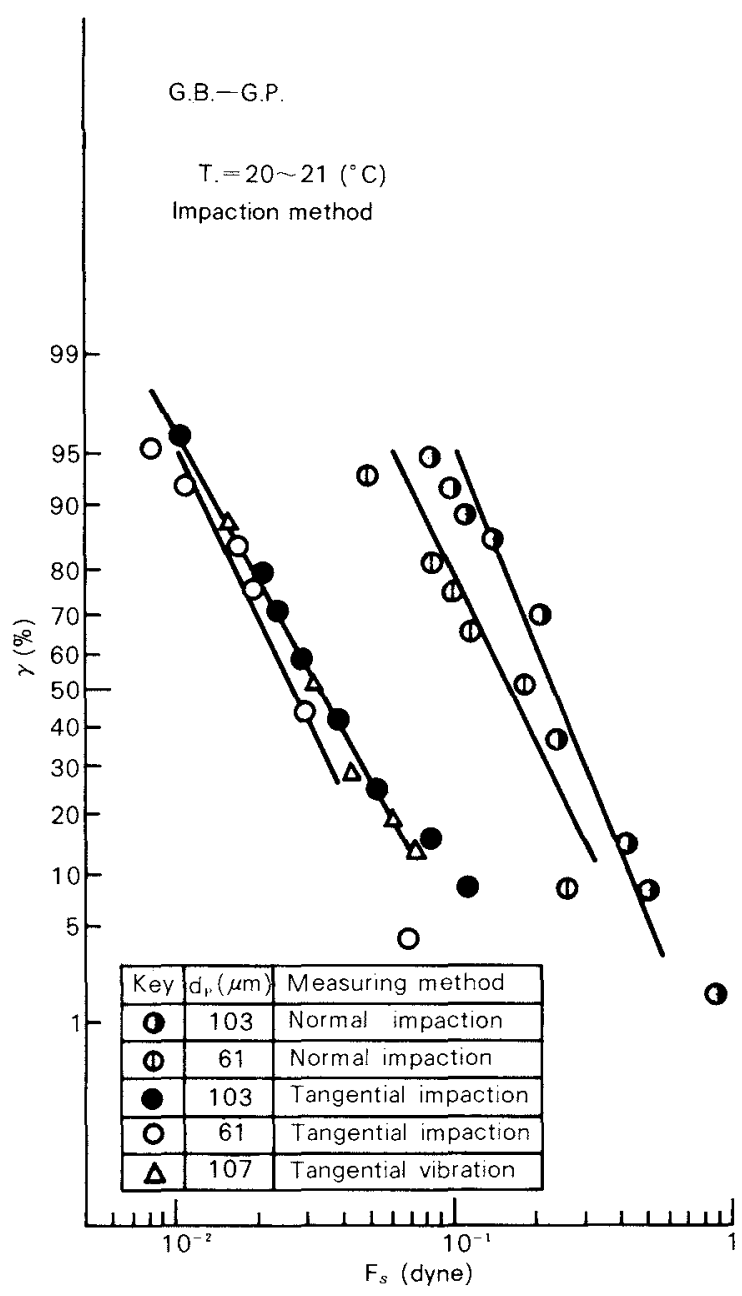

Fig. 8 The results of impaction separation method Comparison of normal and tangential separation

and $6.1 \mu \mathrm{m}$ for the particles of $103 \mu \mathrm{m}$ and $61 \mu \mathrm{m}$ in diameter respectively. These results are much larger than those estimated from Hertz's theory mentioned in 3.4 (1) but correspond to the roughness of solid surface observed by a microscope. And it must be pointed out that the difference between these two radii of contacting plane of two particles with different diameter is much smaller than the differences of the values calculated and estimated with equation (6) and by Dahneke, who took account of the effect of adhesion force between a particle and a plane surface. At this stage of investigation, it can be supposed from these results that the contacting conditions determined by the roughness of the glass surface which does not depend upon particle size is more important than those caused by the deformation of the particle on the contacting plane depending upon the particle size. 


\section{Measurement of adhesion force at high tem- perature}

\section{1 Measuring method}

For the vibration method, a heater was attached to the back side of the adhesion plate. As increase of the adhesion force was expected at the higher temperature, a tangential separation method requiring small separation force was adopted in the temperature range up to about $350^{\circ} \mathrm{C}$. In the impaction method, on the other hand, the tapping was conducted in a small oven covering the whole measuring cell, which performed the measurement up to about $460^{\circ} \mathrm{C}$.

\section{2 Results and discussions}

The effect of temperature on the separation force by the tangential vibration method and by the tangential impaction method are shown in Figs. 9 and 10 respectively.

The effect of temperature on the average value of separation force of glass beads is shown in Fig. 11, where the following tendency can be seen. The adhesion force reduces with increasing temperature to reach the minimum value around $100^{\circ} \mathrm{C}$. After that it continues to in-

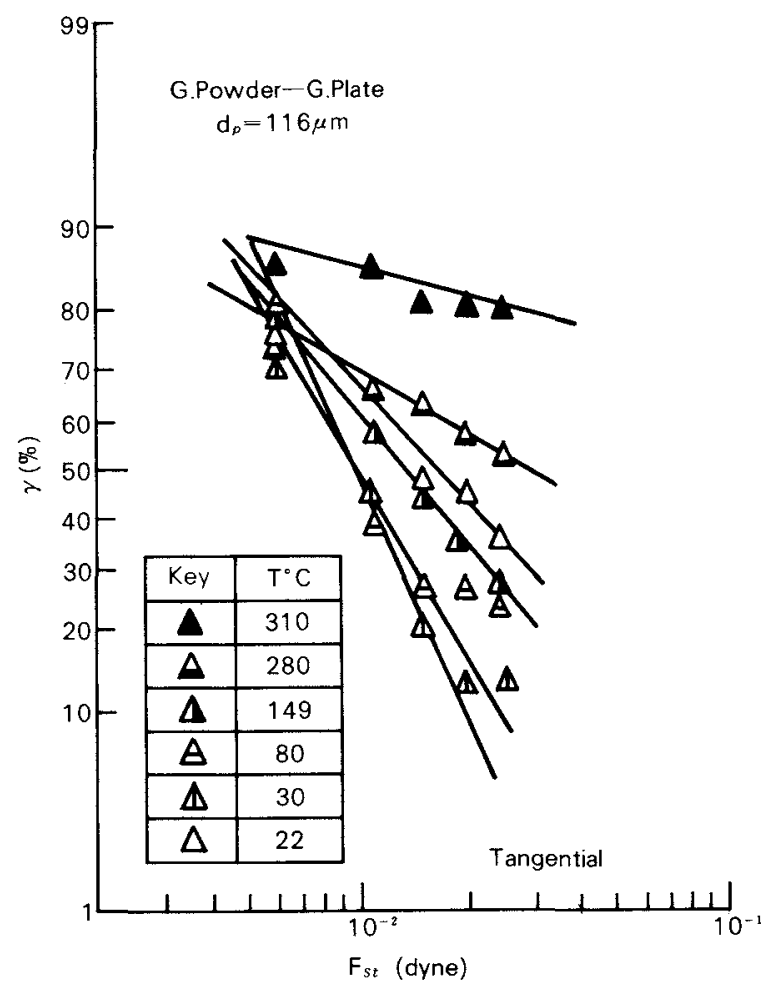

Fig. 9 The effect of temperature on the separation force by vibration tangential separation method

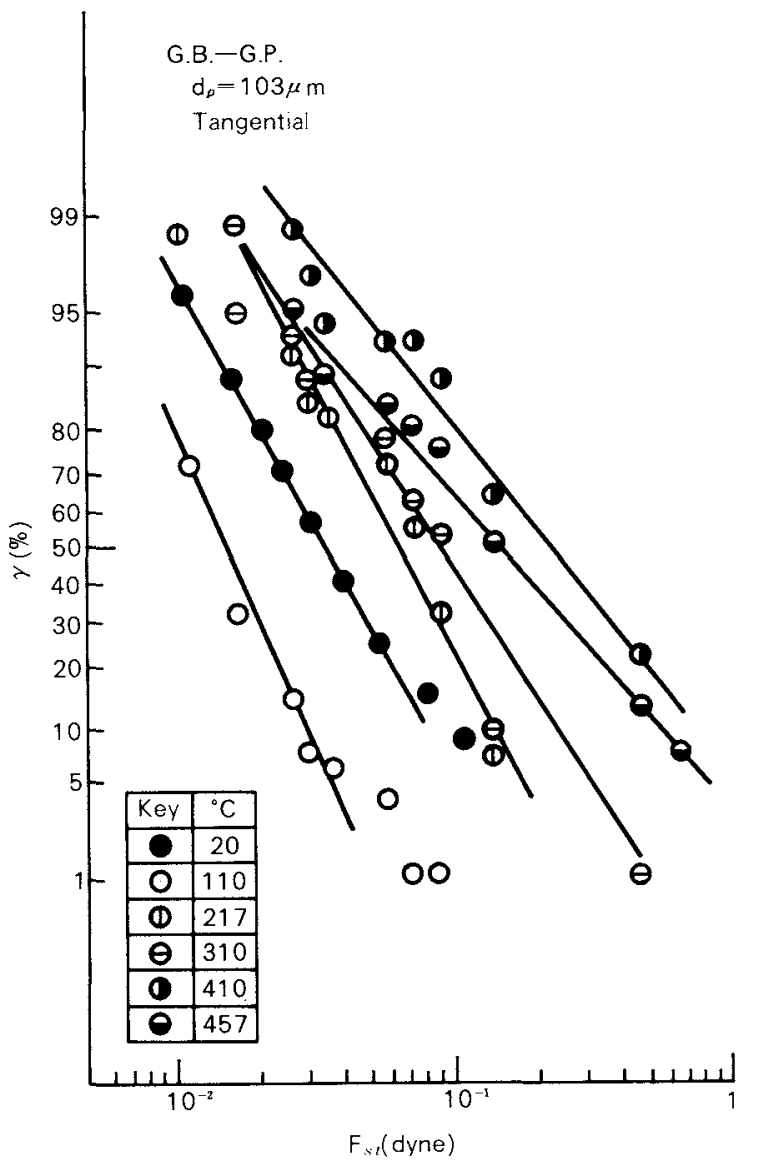

Fig. 10 The effect of temperature on separation force of glass beads by impaction tangential method

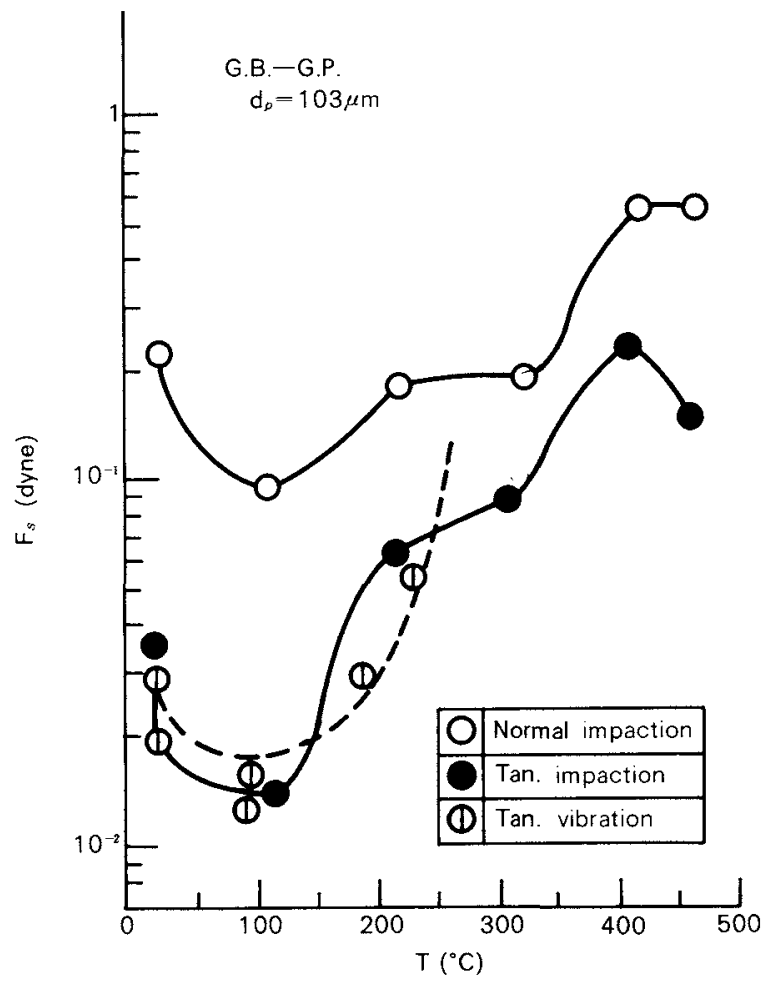

Fig. 11 The effect of temperature on the average value of separation force of glass beads. 
crease with rising temperature to exceed the value of the room temperature in the range between about 200 and $300^{\circ} \mathrm{C}$. At about $400^{\circ} \mathrm{C}$, it shows a few times as large value as that of the room temperature and does not seem to increase at the higher temperature. The tendency of the adhesion force having the minimum value at about $100^{\circ} \mathrm{C}$ can be regarded as a general one, as it is seen with the material such as silica sand and FCC catalyst particles. It was also generally admitted that the adhesion force increases considerably with further rising temperature.

The detailed comparison of the data by the normal impaction method with those by the tangential one made clear that the difference between them reduced with the increasing temperature. Using equation (7), it was found that the radius of contacting plane $a$ increased from $7.7 \mu \mathrm{m}$ at room temperature to $23 \mu \mathrm{m}$ at $310^{\circ} \mathrm{C}$.

In this way, an experimental explanation has been given to realize the mechanism for the change of adhesion force by the indirect estimation of the deformation of the contacting plane from the separating mechanism of particles.

\section{Conclusions}

To make clear the problems in the measurement of adhesion force of single particles of powder and to extend the measurement range, new measuring equipments were developed, which also made possible the measurement under special conditions such as high temperature. From these results, the following points were made clear.

(1) For the measurement of adhesion force of single particles, a vibration separation method and an impaction separation method have been developed and proved to be useful methods.

(2) It was found that the rolling of particles gives remarkable effect on the separation in these methods. A fundamental equation was derived based on the theoretical consideration of the separating mechanism with rolling of particles.

(3) Additionally, a tengential separation method making use of the rolling action of particles has been developed and it has been shown that useful informations about the contacting condition and separating mechanism of a particle can be obtained from comparison of the tangential and the normal separation method.

(4) The applicability of the above method to the measurement at high temperature was confirmed and it was found that the adhesion force of glass beads reduced with increasing temperature up to $100^{\circ} \mathrm{C}$ and then continued to increase.

\section{Acknowledgements}

This research was supported by the Asahi Glass Foundation for Industrial Technology in 1978 and 1979, and by a Grant-in-Aid for Scientific Research from the Ministry of Education, Science and Culture, Japan in 1979 and 1980 (No. 443024).

\section{Nomenclature}

$a:$ radius of contact circle

$d_{p}$ : diameter of particle

$E$ : Young's modulus

$f_{a d}:$ adhesion force per unit surface

$F_{a d}$ : separation force of a particle to normal direction

[ $\mu \mathrm{m}$ or $\mathrm{mm}]$

$\left[\mathrm{kg} / \mathrm{mm}^{2}\right]$

[dyne $/ \mathrm{cm}^{2}$ ]

$F_{s}:$ separation force

[dyne]

[dyne]

$\bar{F}_{s}: F_{s}$ at $\gamma=50 \%$

[dyne]

$F_{s n}$ : normal component of separation force [dyne]

$F_{s t}:$ tangential component of separation force [dyne]

$H$ : adhesion force of a single particle [dyne]

$m$ : mass of a particle

[g]

$\alpha$ : amplitude of vibration

[cm or $\mathrm{mm}]$

$\gamma:$ residual percentage of particles

$\nu$ : Poisson's ratio

$\rho_{s}:$ density of particle

$\sigma:$ standard deviation

$\varphi$ : inclination of plane surface

$\omega$ : angular velocity of vibration $[\% / \mathrm{sec}$ or $\mathrm{rad} / \mathrm{sec}]$

\section{References}

1) Jimbo, G.: J. Soc. Material Sci., Japan, 16, 291 (1967).

2) Asakawa, S. and G. Jimbo: ibid, 16, 358 (1967).

3) Jimbo, G., R. Yamazaki, G. Hong and H. Hirano: 11 th Fall Meeting of Soc. Chem. Eng., Japan, Preprint, p.445 (Tokyo 1977).

4) Yamazaki, R., Y. Imai, G. Hong and G. Jimbo: 12th Fall Meeting of Soc. Chem. Eng., Japan, Preprint p.335 (Tokyo 1978).

5) Jimbo, G. and R. Yamazaki: European Symposium Particle Technology 1980, Preprint p.1064 (June 3-5, 1980, Amsterdam).

6) Polke, R.: Chemie - Ing. - Technik, 40, 1057 (1968).

7) Dahneke, B.: J. Colloid \& Interface Sci., 40, 1 (1972). 